Introduction Autonomic Nervous System allows a proper response to different environmental stimuli and is crucial for worker to adapt to physical effort and psychological stress i.e. shift-work, environmental temperature changes, long-lasting gravitational stimulus, physical demand.

Methods ECG, beat-by-beat Blood Pressure and Respiration are recorded while supine and during gravitational stimulus ( $75^{\circ}$ Head-up tilt). Sinus Arrhythmia and Valsalva Manoeuvre provide the relationship between respiratory activity and heart rate and the integrity of baroreceptors functioning. Hyperventilation, mental arithmetic, exposure to noise or occupational pollutants may also be used.

ECG and BP are recorded during working and sleeping time over 24 hours or more.

Spectral analysis provides indexes of cardiovascular autonomic control. From RR variability, Low Frequency $\left(\approx 0.1 \mathrm{~Hz}, \mathrm{LF}_{\mathrm{RR}}\right)$ and High Frequency $\left(\approx 0,25 \mathrm{~Hz}, \mathrm{HF}_{\mathrm{RR}}\right)$ oscillatory components are indexes of cardiac sympathetic and vagal modulation, respectively. LF/HF quantify the instantaneous cardiac sympatho-vagal tone. From systolic arterial pressure (SAP) variability, Low Frequency oscillatory component $\left(\approx 0.1 \mathrm{~Hz}, \mathrm{LF}_{\mathrm{SAP}}\right)$ is an index of vascular sympathetic modulation. Baroreflex sensitivity are assessed in the time (BRSseq) and frequency ( $\alpha L F)$ domain.

Symbolic analysis of heart period may furnish additional non-linear tools to quantify the sympatho-vagal modulation to the heart.

Results In susceptible workers, triggers involving autonomic nervous system, potentially present in the work environment (mental, visual, visceral or orthostatic stress) may promote neuro-mediated syncope or orthostatic intolerance syndromes.

Hypertensive workers show an early cardiovascular sympathetic predominance with 24 hour sympatho-vagal balance impairment

In healthy shift workers a blunted circadian oscillations of heart rate and $\mathrm{LF} / \mathrm{HF}$ during morning and night shift may represent a possible early sign of misadaptation to shift work in absence of any disease.

Discussion The assessment of individual cardiovascular autonomic profile in clinical laboratory and during working activity may help prevention of cardiovascular disease and promote health and safety at work by a 'Precise Medicine' approach.

\section{DEVELOPMENT OF EATING PATTERN FOR PEOPLE WITH UNCONTROLLED HYPERTENSION USING FAMILY PARTICIPATION APPROACH}

${ }^{1}$ B Duangduen*, ${ }^{2}$ A Sivaporn, ${ }^{2}$ B Waraporn. I'Instructor, Chiang Mai University, Chiang Mai, Thailand; ${ }^{2}$ Assistant Professor, Chiang Mai University, Chiang Mai, Thailand

\subsection{6/oemed-2018-ICOHabstracts. 175}

Introduction Eating is essential for controlling blood pressure level among hypertensive worker, therefore it is important that eating patterns should suit the context and lifestyle of these persons.

Methods A developmental research design was used to conduct this study. This study aimed to develop eating patterns for hypertensive worker with uncontrolled hypertension using family participation based on the family participation concept from related literature reviewed and to study the feasibility of using the eating pattern in real situations. The population in the study consisted of:

- hypertensive worker with uncontrolled hypertension $(\mathrm{N}=68)$,
- their family members $(\mathrm{N}=68)$, and

- Healthcare volunteer villages and Health promoting hospital staff responsible for chronic illness $(\mathrm{N}=11)$.

The study instruments were comprised of:

- People with Uncontrolled Hypertension Eating Questionnaire,

- Family Members Eating Questionnaire,

- Focus Group discussion guide for Village Healthcare Volunteers and Health promoting Hospital Staff,

- Feasibility of Using the Eating Pattern in Real Situation Questionnaire, and

- Developmental Plan of the eating pattern for people with uncontrolled hypertension using family participation approach.

The data were analysed using descriptive statistics and qualitative analysis.

Results Two main results were found. The data from situation analysis phase documented that the workers still had improper eating behaviour and their main obstacle for improving their eating behaviour is time and their socioeconomic status. Secondly, the new eating pattern were comprised of three main ideas: increasing consumption of vegetables, following a low salt diet criteria, and appropriate eating. And the result of possibility for using new eating pattern showed the new plan was suitable for the lifestyle of worker with the uncontrolled hypertension and their family members at a moderate level $(x=2.50$, S.D. $=0.62)$ and was feasible $(x=2.47$, S.D. $=0.61)$. In addition, the worker suggested that it is important to use mass media to provide more information about eating patterns to highlight the future benefits for uncontrolled hypertensive persons. Communities should encourage this group and their family members to use the eating plan and promote this eating pattern in the community and in the future, the eating plan should include recipes for hypertensive that use local vegetables known to lower high blood pressure. These recipes should be easy for to prepare.

Conclusion In conclusion, although this study could explore the eating situation of the worker with uncontrolled hypertension and can created the new eating plan, but for future study, we should to study more about how to improve the uncontrolled hypertension work eating behaviour following this finding.

\section{CARDIOVASCULAR REACTION TO STRESSFUL SITUATIONS IN PUBLIC TRANSPORT DRIVERS}

J Siedlecka*, E Gadzicka, T Makowiec-Dąbrowska, A Bortkiewicz. Nofer Institute of Occupational Medicine, Lodz, Poland

\subsection{6/oemed-2018-ICOHabstracts.176}

Introduction The job of a bus driver in public transport is characterised by an increased risk of cardiovascular diseases, including hypertension. The present project has been focused on investigating the cardiovascular response to professional duties and work-related stress in public transport drivers with treated and untreated arterial hypertension vs the drivers with normal blood pressure values.

Methods The study group were 61 drivers aged 37-58 years. Thirty of them had a hypertension: 15 subjects received systematic treatment and the other 15 had no hypotensive therapy. Normal BP values were found in 31 subjects. All the subjects had general medical examination, responded to a questionnaire 
regarding the risk factors and symptoms of hypertension, had 24 hour heart rate and blood pressure monitoring. The subjects were asked to write all the stressful situations at work while the monitoring of the haemodynamic functions was proceeding.

Results Seventy-five conditions with different level of stresogenicity were identified and appropriate BP and HR values from the monitoring records were assigned. The statistical methods included analysis of variance and logistic regression model. The results revealed that in subjects with untreated arterial hypertension, the cardiovascular response to stressogenic conditions consisted in a higher increase in systolic BP $(180 / 113 \mathrm{~mm} \mathrm{Hg})$ than in those with normal BP $(144 / 94 \mathrm{~mm} \mathrm{Hg})$ or receiving hypotensive treatment $(153 / 101 \mathrm{~mm} \mathrm{Hg}) ;(p<0.01)$.

Discussion The bus drivers' job was characterised by a high level of work-related stress. Therefore, it's necessary to undertake preventive measures to reduce the level of stress, e.g. by training in stress coping and conflict solving strategies and to periodically perform long-term monitoring of arterial blood pressure in the workers at risk. The study points to a high significance of the hypotensive therapy which, when combined with a healthy lifestyle, ensures a better tolerance of stressogenic conditions of the bus driver's job.

\section{HIGH CARDIOVASCULAR RISK IN BELGIAN WORKERS: PREVALENCE ACROSS ECONOMIC SECTORS}

${ }^{1}$ GJM Vandersmissen*, 1,2L Godderis. 'Group IDEWE, External Service for Prevention and Protection at work, Interleuvenlaan 58, 3001 Heverlee, Belgium; ${ }^{2}$ Katholieke Universiteit Leuven, Occupational, Environmental and Insurance Medicine, Kapucijnenvoer 35/5, 3000 Leuven, Belgium

\subsection{6/oemed-2018-ICOHabstracts. 177}

Introduction Cardiovascular disease (CVD) remains the leading cause of mortality and morbidity in Europe. Addressing the modifiable health factors and health behaviours that are associated with CVD is needed to keep workers at work longer and healthier.

The aim of this study was to assess the prevalence of high cardiovascular risk in Belgian workers and its relationship with demographic characteristics and economic sectors.

Methods A cross-sectional study was conducted on data from 132.199 workers under medical surveillance of group IDEWE, External Service for Prevention and Protection at work in 2016. 55,2\% were male, mean age was 38 years.

Four cardiovascular health metrics (smoking, body mass index, physical activity and blood pressure) were categorised as 'ideal', 'intermediate' or 'poor' according to the American Heart Association (AHA) criteria. A 'high cardiovascular risk' group was defined as those workers meeting 3 or 4 'poor' cardiovascular health metrics.

Results Overall 7\% of workers met 3 or 4 'poor' cardiovascular health metrics and were labelled high cardiovascular risk, $9,2 \%$ of men and 4,3\% of women. The prevalence of high cardiovascular risk increased with age from $3,5 \%$ in the age group under 25 years to $10,1 \%$ in those 55 years or older. Transportation and Construction had the highest prevalence of high cardiovascular risk, $17,3 \%$ and $12,4 \%$ respectively. The lowest prevalence, 3,4\%, was observed in Education. The differences remained statistically significant after adjustment for age and gender.
Conclusion A considerable number of workers are at risk for CVD. Significant differences exist between sectors. Risk factors for CVD are modifiable and the benefits of investing in workplace health promotion are clear. Especially workers in Transportation and Construction could benefit from customised worksite wellness programs. Additional research is needed about the relationship between occupation and cardiovascular risk factors and cardiovascular health.

\section{MORNING WALK AS EXERCISE - VARIATION WITH AGE, SEX AND HEALTH STATUS}

${ }^{1}$ SK Sahoo*, ${ }^{2} \mathrm{JB}$ Sahoo, ${ }^{2}$ Saurabh, ${ }^{3}$ Kritali. ${ }^{1}$ Occupational Physician, Noida, India; ${ }^{2}$ UCommunity Service Advisor, Noida, India; ${ }^{3}$ SCasualty Medical Officer, Apollo Medical Hospital, Noida, India

\subsection{6/oemed-2018-ICOHabstracts. 178}

Introduction Exercise is the best risk and health modulator in Industrial setup but is ill-used.

The purpose of the study is to find out, the influence of age, sex, health status, changes in blood pressure, various metabolic parameters (glucose and lipids), consequent to morning walk used as a tool of exercise vis-à-vis ICMR (Indian Council of Medical Research) reference man and woman.

Methods It is prospective study conducted on 58 males and females each, with active work from 1 st Jan 2017 to 30th Jun 2017. The reasons for variation in systolic blood pressure, diastolic blood pressure, BMI, fasting blood sugar, Post prandial blood glucose, total cholesterol, HDL Cholesterol, LDL Cholesterol, VLDL Cholesterol are collated. The data is analysed using mean, standard deviation and percentage.

Results The mean age of men is $(n=58) 42.34 \pm 15.27$ and the mean age of women is $43.06 \pm 9.77$. Findings are shown in table 1 .

\begin{tabular}{lll} 
Abstract 1359 Table 1 & \\
\hline & Men & Women \\
\hline BMI & $25.9 \pm 4.49$ & $27.32 \pm 3.77$ \\
HDLC & $40.86 \pm 8.86$ & $45.72 \pm 13.4$ \\
LDLC & 100.67 & 111.01 \\
& \pm 30.70 & \pm 35.29 \\
VLDLC & $27.81 \pm 17.01$ & $31.29 \pm 18.32$ \\
TC & 163.43 & $185 \pm 35.13$ \\
& \pm 33.63 & \\
FBS & $99.94 \pm 30.19$ & 102.60 \\
& & \pm 33.27 \\
PPS & 123.20 & 127.05 \\
& \pm 17.80 & \pm 54.83 \\
SBP & $123.13 \pm 178$ & 123.93 \\
& & \pm 16.87 \\
DBP & $75 \pm 8.2$ & $79.51 \pm 10.43$ \\
\hline
\end{tabular}

Conclusion Exercise has more beneficial effects in man compared to woman. It influences systolic blood pressure more than the diastolic blood pressure. Effects on HDL Cholesterol is more in woman than in men. Other cholesterol parameters, like LDL and VLDL and Total cholesterol has more impact on men compared to woman. On glucose parameters, it has equal responses. 